\title{
Análisis Espacial de los Hogares con Jefaturas Femeninas en Ciudad J uárez, Chihuahua, México
}

\author{
Spatial Analysis of Female-Headed Households in Ciudad J uarez, Chihuahua, \\ Mexico
}

\author{
Denisse Yssel Ortega Leal \\ Universidad Autónoma de Ciudad Juárez - México \\ dyol_87@hotmail.com \\ Vladimir Hernández Hernández \\ Universidad Autónoma de Ciudad Juárez - México \\ dyol_87@hotmail.com
}

\section{Resumen}

El objetivo de este artículo es identificar empíricamente los agrupamientos geográficos de hogares con jefaturas femeninas y valorar su relación con el rezago social mediante el uso de análisis exploratorio de datos espaciales. La información fue obtenida de los Censos de Población y Vivienda de los periodos 2000 y 2010. A través del Índice de Morán (IM) y los indicadores locales de asociación espacial (LISA) fue posible determinar valores significativos de autocorrelación espacial a nivel intra-urbano. En general, se probó la utilidad del método de investigación, pero se cuestiona la naturaleza de la información proveniente de los datos oficiales ya que estos no capturan diferencias en la variable de hogares con jefaturas femeninas. .

Palabras-Clave: Jefaturas Femeninas; Rezago Social; Análisis Espacial; Auto Correlación Espacial Negativa; Análisis Exploratorio de Datos Espaciales (AEDE).

\begin{abstract}
The objective of this article is to identify geographic clusters of female-headed households and evaluate its relationship with the social gap by using exploratory spatial data analysis. The information was obtained from the Census of Population and Housing of two periods 2000 and 2010. Through the Moran Index (IM) and Local Indicators of Spatial Association (LISA) it was possible to determine significant values of spatial autocorrelation at the intra-urban level. In general, the utility of the research method was tested, but the nature of the information from the official data is questioned because they do not capture differences in femaleheaded households variable.
\end{abstract}

Keywords: Female-Headed Households; Social Gap; Spatial Analysis; Negative Spatial Autocorrelation; Exploratory Spatial Data Analysis (ESDA). 


\section{Introducción}

La importancia de los temas de género ha tomado relevancia en el debate social y económico a nivel mundial. Temáticas como la feminización de la pobreza representan una discusión prioritaria ya que este fenómeno prevalece no sólo en los países desarrollados; sino también en países subdesarrollados (LIMAS, 2010; JANSON, 2010, SOARES, 2011; CAMPOS, 2011; CASTILLO GALLARDO, 2011). Dado esto y en relación a las mujeres jefas de familia, se tiene argumentos que sostienen que una supuesta condición de vulnerabilidad propician niveles de rezago más elevados entre los hogares liderados por mujeres. (ARIZA y DE OLIVEIRA, 2001)

En el contexto de Ciudad Juárez, adyacente a la frontera con los Estados Unidos y debido a la fuerte dependencia de la primera con la economía estadounidense, los impactos en las pérdidas de empleo suelen ser más severas. Por otra parte, el modelo de industrialización que se impulsó desde la década de los años sesenta del siglo XX tiene aún vigencia, $\mathrm{y}$ se conoce como industria maquiladora, donde sobresale la ocupación de mano de obra femenina.

El empleo femenino dentro de la Industria maquiladora ha sido parte fundamental de la economía local. Tanto así que para el año de 1991 en Ciudad Juárez el 39.8 por ciento de la población empleada en el sector maquilador eran mujeres entre los 15 y 19 años de edad; si bien el porcentaje es elevando, al considerar al siguiente grupo de edad la cifra aumenta. El grupo de edades entre 20 y 24 años la el porcentaje incrementaba a 44.9 por ciento (CARRILLO, 2001). Ciudad Juárez se consolidaba como una de las ciudades fronterizas con mayor auge económico a nivel nacional. Sin embargo, fue hasta el año 2002 cuando se registró el mayor porcentaje de desempleo con una tasa del 2.8 por ciento ocasionando incrementos importantes en los niveles de pobreza de la ciudad.

Un estudio previo (LIMAS, 2010) informaba sobre un incremento de la población pobre femenina en ciudad Juárez. Se esbozaba que el problema radicaba en la situación de vulnerabilidad de las mujeres, debido a su condición de género, dando como resultado la feminización de la pobreza. Ahora bien, se tienen antecedentes de que los niveles de pobreza en ciudad Juárez están aumentando y que las condiciones de vulnerabilidad colocan a las mujeres y en especial a las jefas de familia en una situación desventajosa. El problema es que no tenemos un trabajo que permita identificar patrones espaciales que en consecuencia, sirva como un instrumento para la generación de políticas enfocadas a este u otros grupos de la población.

En términos empíricos este trabajo propone hacer uso de las técnicas de análisis exploratorio de datos espaciales como una metodología auxiliar en la detección de agrupamientos espaciales; en consecuencia, favorecer la focalización de los programas sociales.

\section{Hogares con Jefaturas Femeninas y Rezago Social}

Ante los cambios en la estructura económica y la continua inmersión de las mujeres al mercado laboral, se ven transformados los roles tradicionalmente fijados entre mujeres amas de casa, en los que incluso éstas asumen completamente el rol de proveedoras. En México, algunos estudios mencionan que la mayor parte de mujeres jefas de familia son jefas por viudez (39.3\% en zonas urbanas, $54.5 \%$ en zonas rurales); le sigue la causa de separación y divorcio (34.7\% en zonas urbanas y $36.8 \%$ en zonas rurales). (LÁZARO, ZAPATA, 
MARTÍNEZ, ALBERTI, 2005).

El porcentaje mayor de los ingresos en hogares con jefaturas femeninas en las zonas urbanas va destinado a gasto en alimentos. (LÁZARO, ZAPATA, MARTÍNEZ, ALBERTI, 2005). El 94 por ciento de las mujeres realizan, por lo menos, doble jornada de trabajo. (ROSA LÁZARO, EMMA ZAPATA y BEATRIZ MARTÍNEZ, 2005).

Al respecto Buvinic 1991, se refiere a la fuerte relación entre hogares pobres $\mathrm{y}$ jefaturas femeninas y esboza a la feminización de la pobreza como uno de los problemas mundiales emergentes. Igualmente Limas (2010), encuentra que la conjugación de dos fenómenos negativos: pobreza $y$ desigualdad basadas en la condición de género generan la pobreza en hogares de jefatura femenina. (LIMAS, 2010).

Algunos autores comparten la idea sobre el hecho de que el creciente número de mujeres trabajadoras - proveedoras de ingresos y tomadoras de decisiones; mismas que conforman el grueso de los hogares con jefaturas femeninas en México, reciben bajos salarios a la misma vez que encuentran serias dificultades para incorporarse a una estructura de oportunidades.

De acuerdo a autoras como Barquet (1997), y Acosta (1994) el trabajo especialmente de las mujeres - en los espacios doméstico y extra doméstico, para la obtención de ingresos monetarios y no monetarios, no son lo suficientemente bien remunerados para garantizar la supervivencia y la reproducción familiares. De acuerdo con lo anterior el bajo nivel de ingresos afecta negativamente al género femenino y da como consecuencia se genera una mayor vulnerabilidad económica.

Se tiene por un lado la creciente complejidad del fenómeno de la feminización de la pobreza, y por otra parte el existente vacío de las políticas de género sobre las políticas públicas en las zonas urbanas. Es por eso que la importancia de establecer la relación entre la estructura urbana- la redistribución del ingreso y el género como categoría de análisis; permitiría a su vez conocer aspectos como las desigualdades de infraestructura y servicios que condicionan la ciertos sectores de población y el tratamiento a las injusticias que sufren los grupos vulnerables.

\section{Método}

El método propuesto para este trabajo hace uso de las técnicas exploratorias de datos espaciales. Los datos para realizar el análisis provienen de los Censos de Población y Vivienda para los años de 2000 y 2010 (INEGI). Las variables usadas en el análisis son el porcentaje de hogares con jefaturas femeninas y el índice de rezago social ambas a nivel de AGEB. El enfoque central del trabajo se esbozó desde la perspectiva multidimensional que desarrolla a la par de la medición del bienestar a través del ingreso y el rezago en los accesos a los derechos sociales. Consideramos que este es uno de los aportes del trabajo, además de construir el Índice de Rezago Social (IRS) y valorar su naturaleza geográfica; es establecer la relación con la distribución de los hogares con jefaturas femeninas. Por lo tanto este trabajo incorporar a los estudios de pobreza y género el análisis espacial de forma explícita

\section{Elaboración del Índice de Rezago Social}

Para le elaboración del Índice se rezago social se utilizó la metodología de CONEVAL (2007) mismo que contempla cuatro rubros que son: educación, salud, calidad y espacios de la vivienda, servicios básicos de la vivienda y activos en el hogar. El índice de rezago social se construye como una suma ponderada de los diferentes indicadores con el objetivo es encontrar un 
modo de condensar la información contenida en un número de variables originales en un conjunto más pequeño de variables (factores) con una pérdida mínima de información. ${ }^{1}$

\section{Análisis Exploratorio de Datos Espaciales (AEDE)}

La importancia del análisis espacial radica en su uso para la toma de decisiones en programas de intervención focalizados $y$ contribuir en la priorización de los recursos. Esta investigación propone el análisis espacial y específicamente la detección de conglomerados geográficos, como técnica útil para la ubicación de hogares con altos índices de rezago social.

El análisis exploratorio de datos espaciales (AEDE) es un conjunto de técnicas que nos permiten relacionar los datos, a través de métodos estadísticos y gráficos se pueden a llegar a descubrir patrones. Una de sus funciones más utilizadas es su potencial para identificar y formular hipótesis que no pueden ser consideradas por las teorías. (HERNÁNDEZ, 2012).

Ahora bien la función de localizar los datos en el espacio tiene importantes implicaciones por la forma como se efectúa el análisis estadístico, debido a que los datos pueden implicar dos de los llamados efectos espaciales: dependencia y heterogeneidad. Supongamos que los valores de hogares con jefaturas femeninas (HJF) no son independientes entre sí, es decir, que los valores porcentuales de HJF se parece en algunas zonas de la ciudad, en otras palabras están espacialmente agrupados. Lo anterior se contrapone al supuesto de independencia en la estadística tradicional.

En la literatura consultada (GARCÍA DE LA ROSA, 2011, citando a ANSELIN, 2005) podemos reinterpretar que la dependencia, expresada en el ejemplo de la hipotética agrupación de los $\mathrm{HJF}$, se refiere como autocorrelación espacial. Y la heterogeneidad se refiere a la diferencia espacial, derivada de la singularidad individual de cada lugar. De manera general, se dice que se puede considerar que cualquier indicador que llegue a identificar autocorrelación espacial puede ser considerado un AEDE. (GARCÍA DE LA ROSA, 2011).

\section{Autocorrelación Espacial Global y Local}

La autocorrelación se evalúa a través del estadístico I de Morán, que detecta cuáles áreas circundantes tienen similares $\mathrm{o}$ disimilares atributos (HERNÁNDEZ, 2012). La autocorrelación espacial puede ser definida como la coincidencia de ubicación espacial de valores (ANSELIN, 2005); existe una autocorrelación positiva cuando los valores altos o bajos de una variable tienden a agruparse en el espacio. En el contexto de esta investigación en las áreas urbanas la autocorrelación significa que las AGEBS con valores altos y bajos del índice de rezago social y hogares con jefaturas femeninas están agrupados.

Un valor positivo del estadístico de I de Moran significa que altos valores (del IRS y de HJF) están rodeados por áreas con altos valores de IRS y HJF (alto-alto) o bajos valores están rodeados por áreas de bajos valores (bajo-bajo). Un valor negativo significa que bajos valores están rodeados por altos valores (bajo-alto) o altos valores están rodeados por bajos valores (alto-bajo). Lo anterior, sugiere dos clases de autocorrelación espacial positiva o clusterización (alto-alto y bajo-bajo) y dos clases de correlación espacial negativa (alto-bajo y bajo-alto) (ANSELIN, SYABRI, \& SMIRNOV, 2002).

De esta manera se determinan las agrupaciones llamadas 'Hot spots' y los 'Cold spots' los cuales dado un conjunto de características ponderadas, identifica grupos 
de funciones con valores altos (puntos calientes) y clústeres de funciones con valores bajos (zonas frías) (LAUREN $\mathrm{M}$. SCOTT, MARK V. JANIKAS, 2010). De acuerdo a otro autor los mapas de clústeres muestran la localización de los valores atípicos espaciales y los grupos de valores bajos y altos y la manera en cómo éstos cambian a través del tiempo (JACQUEZ y GREILING, 2003).

La regla de decisión para validar las hipótesis de trabajo a partir del coeficiente de autocorrelación global (I de Morán) es:

H0 $\mathrm{IM}=0$ el coeficiente de autocorrelación global (IM) es igual a cero, por lo que no existe un patrón espacial, se esperaría un patrón aleatorio del índice de rezago social $\mathrm{y}$ de los hogares con jefaturas femeninas.

H1 IM $\neq 0$ el coeficiente de autocorrelación global (IM) es diferente a cero, por lo tanto existe autocorrelación espacial, en consecuencia, es posible afirmar que los resultados obtenidos del índice de rezago social y de los hogares con jefaturas femeninas no se distribuyen aleatoriamente en el espacio geográfico.

Para estimar los agrupamientos intra-urbanos del IRS y HJF (zonas con alto índice de rezago social y altos valores de HJF) se propone utilizar indicadores locales de asociación espacial (LISA por sus siglas en inglés Local Indicator of Spatial Association). Los conglomerados espaciales de
IRS y HJF se identifican como aquellas localidades o conjunto de localidades contiguas o vecinas en las cuales los indicadores locales son significativos estadísticamente.

\section{Resultados y Análisis}

En esta sección se valoran estadísticamente $y$ se muestran cartográficamente los patrones geográficos de los hogares con jefaturas femeninas (HJF) y los índices de rezago social (IRS). En el cuadro 1 se observan los resultados del I de Morán $^{2}$ para los HJF y el IRS. Estos sugieren validar la hipótesis (H1) que consideran la presencia de autocorrelación espacial positiva. Todos los coeficientes cumplen la regla de decisión que indica que $\mathrm{IM} \neq 0$ (Índice de Morán diferente a cero) lo que permite rechazar la hipótesis nula (H0). Los resultados de estos dos indicadores nos muestran que los HJF y el IRS poseen una estructura geográfica que tiende a concentrarse en sus valores altos y valores bajos.

Autocorrelación espacial global de los HJF y el IRS, ciudad Juárez 2000-2010

\begin{tabular}{cc}
\hline Indicador & I deMorán* \\
HJF 2000 & 0.5259 \\
HJF 2010 & 0.5032 \\
IRS 2000 & 0.4871 \\
IRS 2010 & 0.3358 \\
HJF-IRS 2000 & -0.2439 \\
HJF-IRS 2010 & -0.2285 \\
\hline & *pulonte: Elaboración propia con datos de INEGI
\end{tabular}

Fuente: Elaboración propia con datos de INEGI 
En la figura 1 se muestra la distribución de los resultados del agrupamiento de los HJF según la relación espacial que los congrega. Estos pueden agregarse en clústeres de HJF (zonas vecinas con valores similares de porcentajes de HJF) o en outlier de HJF (zonas vecinas con valores disimilares de porcentajes de HJF).

La distribución espacial de los HJF (véase figura 1) muestra importantes cambios en su patrón espacial entre los periodos $2000 \mathrm{y}$ 2010. En la imagen de la izquierda se presentan los conglomerados intra-urbanos con valores altos (zonas en coloración más gris) de HJF ubicados en la zona norte y norponiente de la ciudad en el periodo del
2000, considerada la zona 'centro de la ciudad'.

Esta es una zona consolidada refiriéndonos a su dotación de servicios públicos e infraestructura aparte de ser de las más antiguas, ya que el crecimiento de la mancha urbana se dio al sur - oriente y sur-poniente de la ciudad. Por otro lado podemos observar que los valores más bajos en el porcentaje de HF se encuentran al sur-poniente de la ciudad donde se daban los nuevos desarrollos habitacionales de interés social. Cabe mencionar que las zonas donde se dan los agrupamientos durante este periodo no eran las zonas con mayores ingresos de la ciudad, esto será importante una vez que analicemos

Figura 1

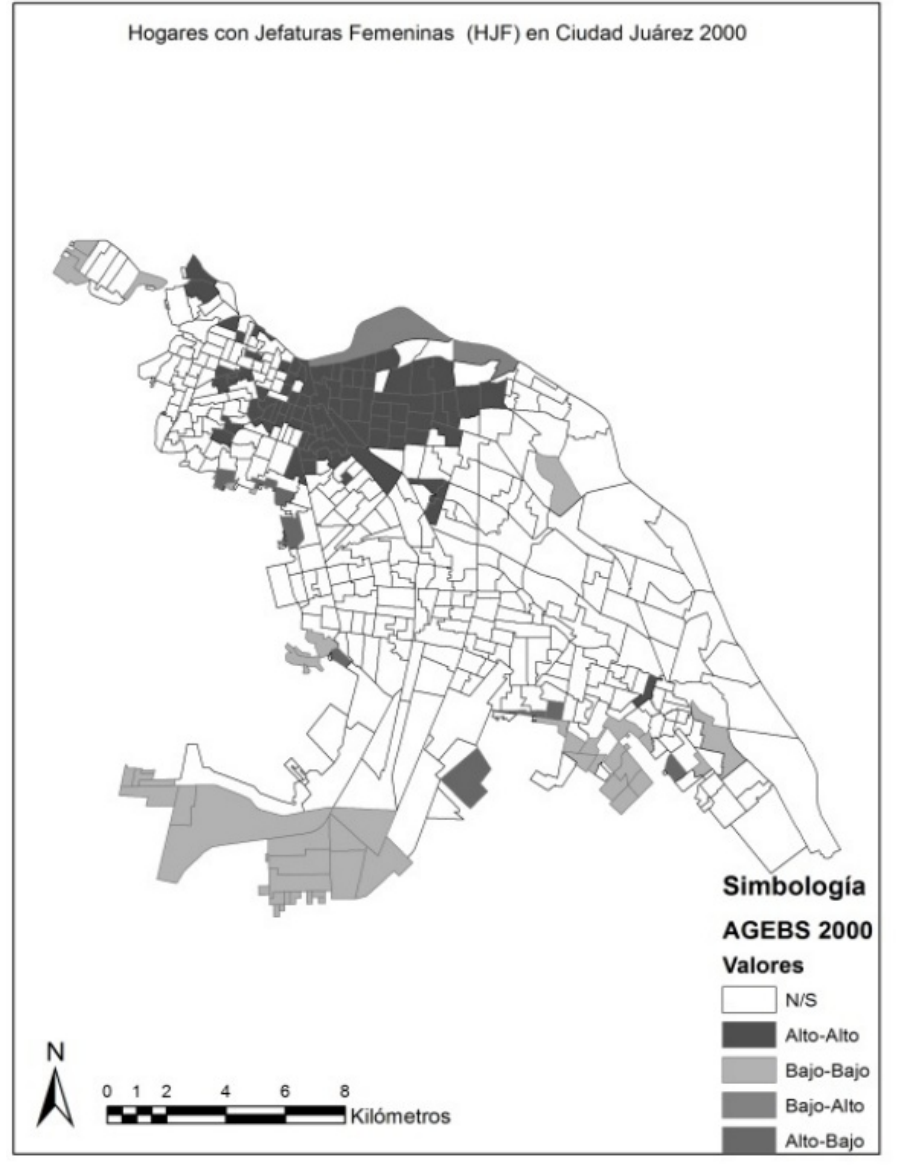

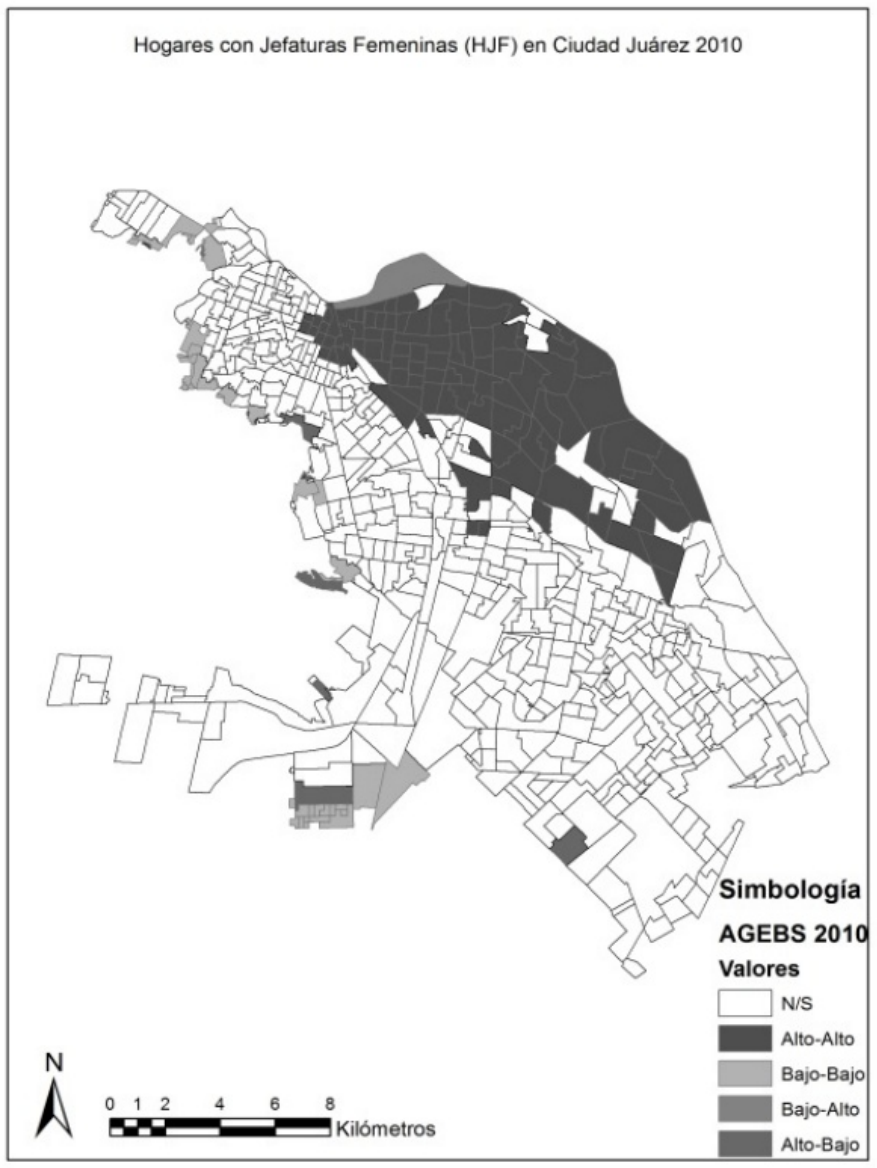


el cambio en el patrón espacial en el periodo del 2010.

En la imagen de la derecha se presenta la distribución de los HF en el 2010. Como podemos observar el patrón espacial se desplazó a las zonas del nororiente de la ciudad, estas zonas están caracterizadas por ser zonas con altos ingresos. Es necesario señalar que esta investigación muestra solo los resultados del análisis exploratorio de datos espaciales cuya materia prima para la obtención de conglomerados intra-urbanos son los datos de INEGI, por lo que nuestras conclusiones estarán limitadas a los resultados que se deriven de la información de esta fuente considerada oficial. En esta imagen podemos observar que en la cantidad de AGEBS que presentan una mayor presencia de hogares con jefaturas femeninas aumentó considerablemente.

La mayor prevalencia de hogares con jefaturas femeninas en zonas con altos ingresos podría sugerir un tipo de jefaturas femeninas que aunque no presentan necesariamente una situación de pobreza o rezago social determinada a partir del ingreso y de indicadores de rezago social, apoya en la descalificación de la hipótesis que afirma las relaciones directas entre hogares con jefaturas femeninas e ingresos menores. A su vez esto estaría indicando que por el contrario los hogares con mujeres a cargo del hogar propician mejores estándares de vida a sus familias. Aunque lo mejor sería que se pudiese tener la relación entre salarios y jefaturas femeninas, no existe la información existente para el periodo del 2010 ya que fue un variable que no se logró captar en el censo del 2010.

En la siguiente figura (figura 2) se muestra la distribución de los resultados del agrupamiento del IRS según la relación espacial que los congrega. Estos pueden agregarse en clústeres de IRS (zonas vecinas con valores similares de porcentajes de IRS) o en outlier de IRS (zonas vecinas con valores disimilares de porcentajes de IRS).

Las áreas con valores bajos (coloración gris tenue) de ISR en el periodo del 2000 se ubican al nororiente y suroriente de la ciudad. El nororiente se caracteriza por ser una zona caracterizada por los altos ingreso, sin embargo las zonas del suroriente en el periodo del 2000 fueron las primeras zonas de crecimiento urbano con vivienda de interés social y es la zona que rodea al aeropuerto, ambas se constituyen como zonas consolidadas en las que el abastecimiento de servicios públicos es muy alta y la densidad poblacional también lo es. Es por eso que son altamente significativas para el software.

Por otro lado, las zonas con mayores índices de rezago social son aquellas áreas que se caracterizan por no tener cobertura total de servicios públicos como luz, agua y drenaje y que aparte de estar ubicadas en áreas circundantes a las sierra de Juárez consideradas como áreas no aptas para el desarrollo habitacional. Otra cuestión sobre estas zonas con alto rezago social es que al estar tan alejadas del centro urbano, los desplazamientos hacia las principales áreas de trabajo como de servicios son largos lo que implica gran uso de tiempo.

Lo que ocurre en el periodo del 2010 es muy similar, solo que en este periodo ya se incorpora todo el nuevo crecimiento urbano desarrollado al suroriente de la ciudad y se ve claramente como tiene valores de rezago social muy bajos. Estas nuevas áreas habitacionales fueron creadas con casi todos los servicios públicos necesarios para sus habitantes. Por su parte las zonas con altos índices de rezago social se conservan pero son menos, e incluyen nuevas zonas que debido a su creciente creación no cuentan aún con servicios básicos. Ante este panorama, se podría decir que las condiciones de rezago social a nivel intraurbano en Ciudad Juárez son mínimas y que la calidad de vida de sus 
Figura 2

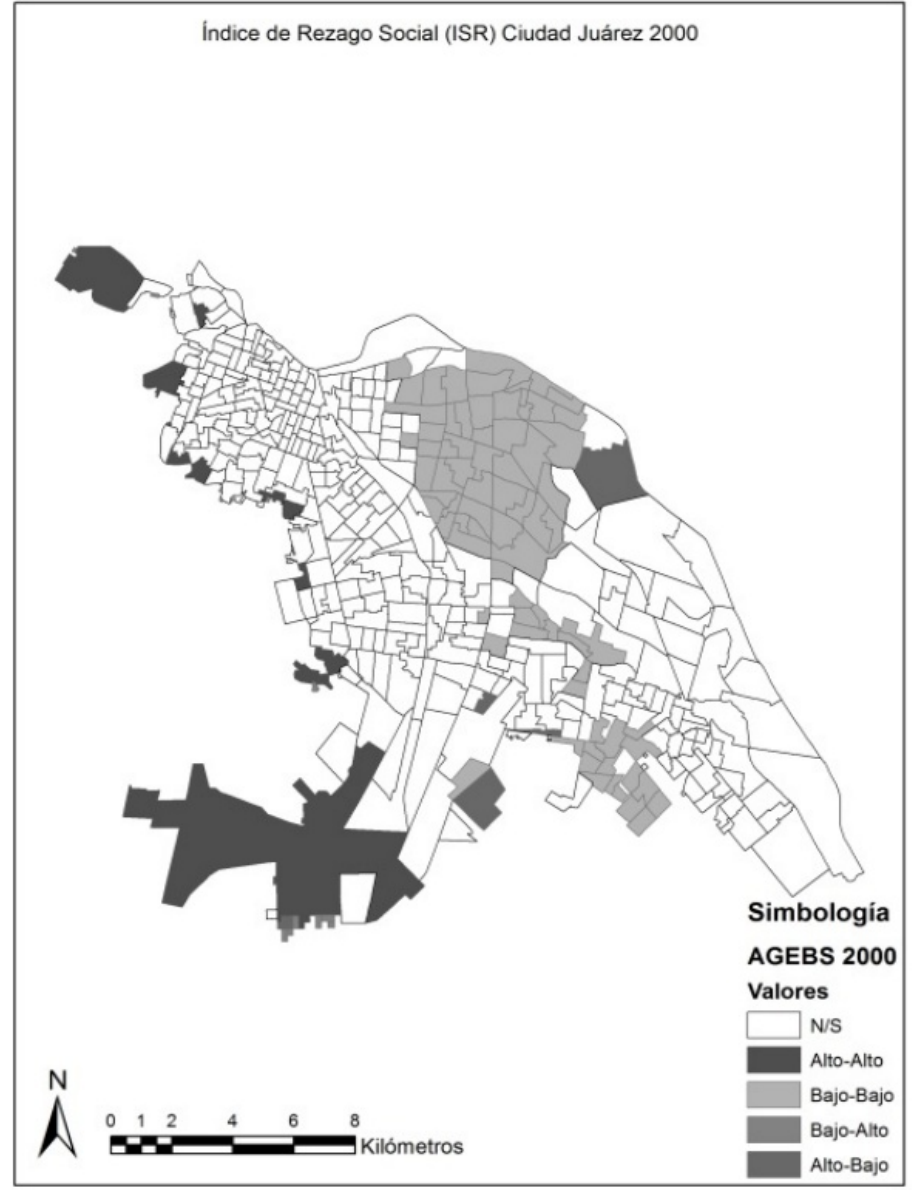

Elaboración propia con datos de INI

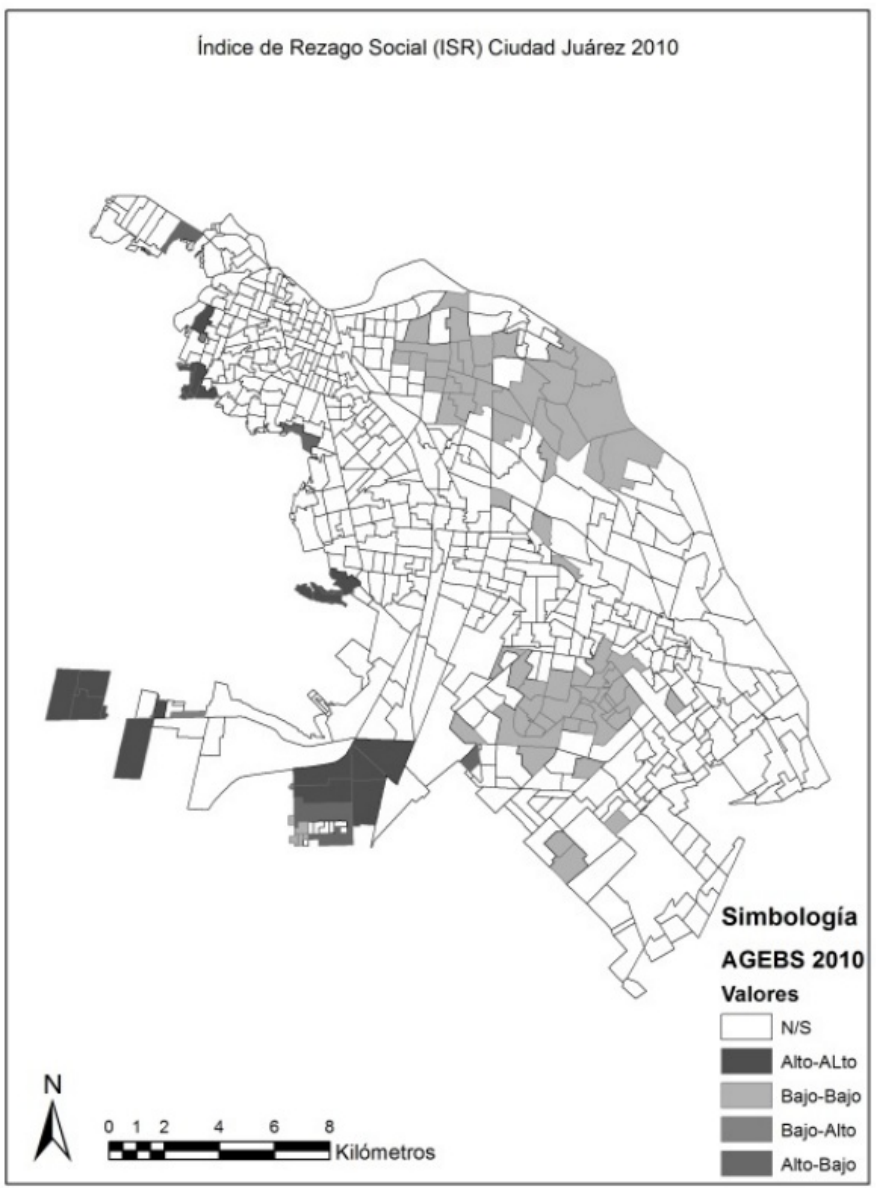

Elaboración propia con datos de INEG habitantes es relativamente alta en comparación con otras áreas urbanas del país.

Como resultado de la relación entre ISR y HF obtuvimos los siguientes mapas, (véase figura 3). Es muy notable el cambio del patrón espacial entre un periodo $\mathrm{y}$ otro. Durante mucho tiempo las preocupaciones de la agenda pública municipal en materia de políticas para el abatimiento y disminución de la pobreza estuvieron dirigidas hacia las zonas que se muestra en coloración más gris en el mapa del 2000.

Los valores más bajos de índice de rezago social en relación con los $\mathrm{HF}$ se encuentran al suroriente de la ciudad en las zonas de nuevo crecimiento urbano. En lo que hay que poner especial atención es en las zonas con valores altos-bajos (AGEBS en color gris medio) ya que como podemos observar estos AGBES se convirtieron después en las zonas con valores altos de rezago social y que presentaban también un importante número de jefaturas femeninas.

En el 2010 a parte de cambiar de un patrón agrupado a un patrón espacial más disperso y ubicado en zonas al sur de la ciudad o bien en las nuevas zonas de crecimiento, estas nuevas zonas presentan situaciones precarias y siguieren AGEBS que presentan altos porcentajes de HF y altos 
Figura 3

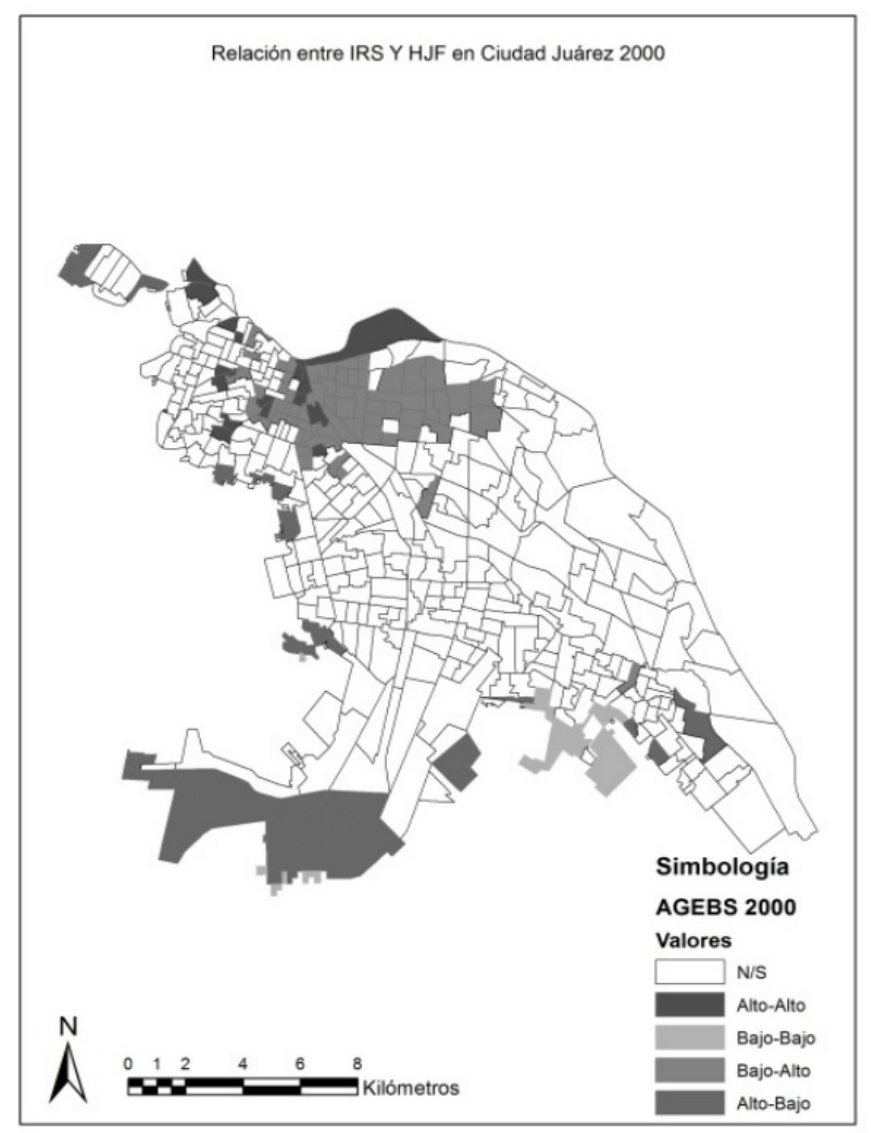

Fuente: Elaboración propia con datos de INEG|

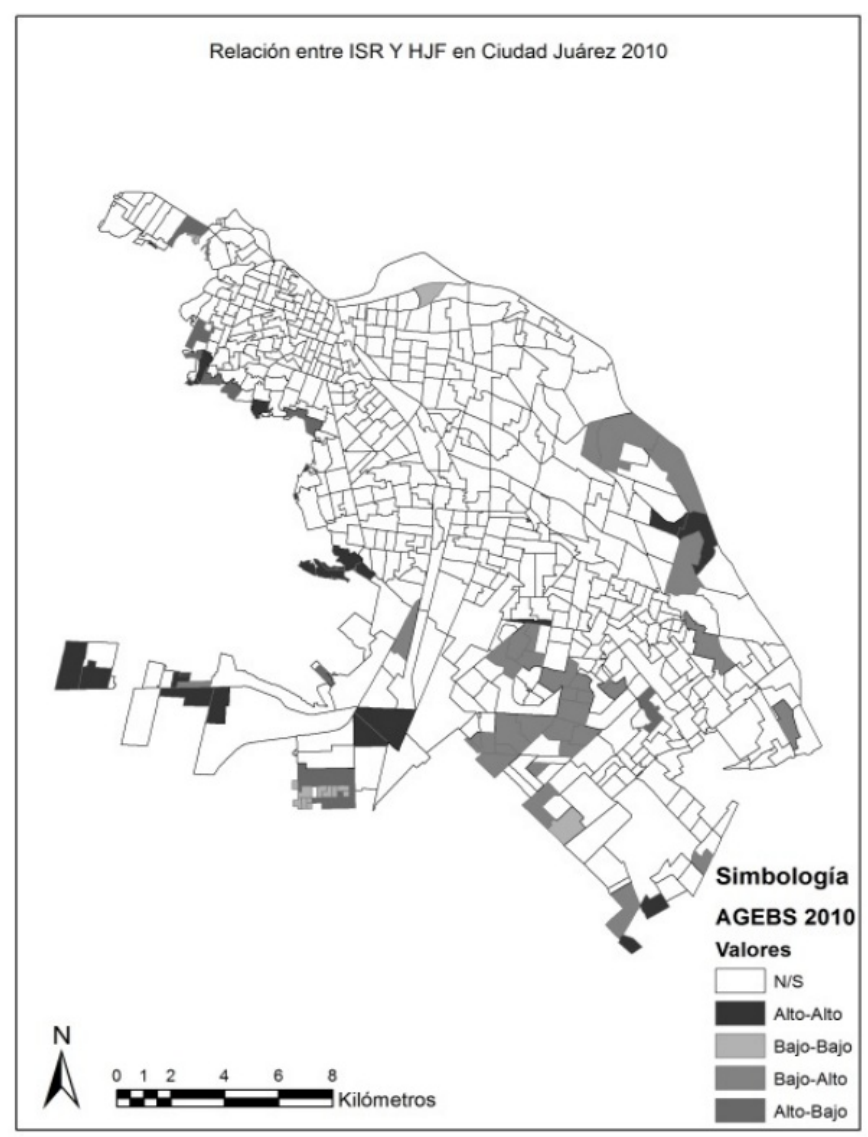

Elaboración propia con datos de INEGI
IRS. Por otra parte los AGEBS con valores bajos-altos (gris medio) abundan en la zona suroriente de la ciudad que indica ser áreas con altos índices de rezago social pero con bajos porcentajes de jefaturas femeninas pero que son lo suficientemente significativas para ser consideradas.

Un patrón espacial de tipo disperso genera un reto importante a la hora de llevar a cabo programas focalizados a la reducción de la pobreza en áreas urbanas ya que es más fácil emprender acciones en áreas agrupadas que en áreas dispersas.

\section{Conclusiones}

El uso de técnicas como el análisis exploratorio de datos espaciales otorga un 'plus' al ubicar un dato censal en el espacio y a escalas más desagregadas lo cual ofrece un buen nivel de fiabilidad ya que se alimenta de datos provenientes de las fuentes oficiales. Con ello se puede tener un mayor conocimiento del contexto espacial para una mejor focalización de programas orientados al desarrollo social de la población en situaciones desfavorables, en las zonas urbanas.

La autocorrelación espacial interpretada a través del Índice de Morán determinó en qué medida las unidades espaciales en nuestro 
caso los AGEBS se asocian unos con otros, teniendo como medida espacial: la distancia. Por otra parte con los indicadores locales de auto correlación espacial (LISA) pudimos establecer los agrupamientos diferenciándolos entre valores altos y bajos.

Sobre los resultados encontrados en el patrón espacial entre aquellas áreas identificadas por tener altos índices de rezago social y altos porcentajes de HJF se muestra evidencia sobre un cambio de un patrón concentrado en 2000 a un patrón más disperso en 2010. En 2010 el patrón esta mayormente disperso entre las zonas habitacionales de reciente creación y hacia donde se ha direccionado el nuevo desarrollo de la ciudad.

Aunque lo anterior señala que se observan importantes cambios en términos de distribución espacial, los índices de auto correlación hablan sobre la existencia de auto correlación espacial negativa. Es decir que aquellos AGEBS que presentan mayor rezago social no se encuentran significativamente asociados espacialmente con los AGEBS con mayores porcentajes con jefaturas femeninas. Dado este resultado sería necesario replantear la hipótesis inicial que afirma que los hogares liderados por mujeres presentan un mayor rezago dada una supuesta condición de mayor vulnerabilidad.

En efecto, al analizar el índice de auto correlación entre AGEBS con alto rezago social y AGEBS con altos porcentajes de jefaturas masculinas se evidencian índices positivos y ligeramente mayores. Con ello emerge una nueva hipótesis que argumentaría que son los hogares con jefaturas masculinas mayormente propensos a niveles de rezago mayores.

Metodologías como la aquí presentada se basan en herramientas que se caracterizan por propiciar un ahorro en tiempos y costos con un buen nivel de desagregación en los datos. Por otro lado permiten conocer aspectos como la tendencia espacial, la heterogeneidad entre las zonas con iguales características a nivel intraurbano y la concentración especial para determinar áreas susceptibles de acción.

Por otro lado las limitaciones que presentan estos métodos es que no reflejan muchos aspectos de las variables en sí. Por ejemplo para el caso de las jefaturas femeninas, los datos censales no ofrecen información como estado civil de la jefa de familia (soltera, viuda o divorciada), criterios como la presencia de los progenitores (si se trata de un hogar monoparental o biparental) o bien criterios como el ingreso (si existen ingresos únicamente femeninos, si hay ingresos tanto masculinos como femeninos) etc.

Desde un punto de vista crítico, dichas limitaciones en la información censal se direccionan para reflexionar sobre la inexistente perspectiva de género en las fuentes de datos oficiales. Ejemplo de ello es que en el concepto de jefe de familia que maneja INEGI se refiere la persona reconocida como tal por los demás integrantes del hogar; puede ser mujer $u$ hombre y supone también que esta persona tiene la autoridad y responsabilidad por los asuntos del hogar y que, en la mayoría de los casos; es el principal apoyo económico. Es decir no hace mucha distinción entre las cualidades del sexo del jefe de familia.

Finalmente, la metodología aquí presentada incorpora una escala de análisis que difícilmente puede captar rasgos esenciales que permitan hacer frente a las desigualdades entre un género y otro hacia situaciones de pobreza más severas. Por lo que el reto tanto de instituciones gubernamentales como de las institutos de investigación consiste en generar datos con sustento en las diferencias de género que permitan realizar acciones de mayor alcance ante problemáticas que emergen a partir de dichas diferencias y que coadyuven a formar 
sociedades más incluyentes y con un alto sentido de equidad.

\footnotetext{
1 Para mayor información sobre la metodología del Índice de Rezago social se puede consultar la siguiente referencia: http://www.coneval.gob.mx/rw/resource/coneval/med _pobreza/1024.pdf

2 La comprobación de la hipótesis se realizó con un nivel de significancia del 95 por ciento, por lo tanto se espera un $\mathrm{p}$-valor $<0.05$.
}

\section{Referencias}

ANSELIN, Luc; SYABRI, Ibnu; SMIRNOY, Oleg. Visualizing multivariate spatial correlation with dynamically linjed windows. 2002. Disponible en: $<$ https://geodacenter.asu.edu/pdf/multi_lisa.p df $>$. Consultado el 27 de Julio de 2014.

ANSELIN, Luc. Exploring Spatial Data with GeoDa. Illinois: Center for Spatially Integrated Social Science, 2005.

BARQUET, Mercedes. Condicionantes de género sobre la pobreza de las mujeres". En: ALATORRE, J. Las mujeres en la pobreza. Cidad del México: Colegio de México, 1997, p. $74-117$.

BUVINIC, Mayra; La vulnerabilidad de los hogares con jefatura femenina: preguntas y opciones de política para America Latina y el Caribe. Serie Unidad mujer y desarrollo. CEPAL, n. 52 p. 7 - 67, 2004.

CARRILLO, Jorge. Mercados de trabajo en la Industria Maquiladora. Tijuana: Plaza y Valdéz editores, 2001.

CASTELLANOS, Rosa Lázaro; MARTELO, Emma Zapata; CORONA, Beatriz Martínez;
; MANZANARES, Pilar Alberti. Jefatura femenina del hogar y transformaciones en los modelos de género tradicionales en dos municipios de Guanajuato. La ventana, $n$. 22, p. 219 - 268, 2005.

CONAPO. (2010, agosto 10). conapo.gob. Disponible en: $<$ http://www.conapo.gob.mx/es/CONAPO/D elimitacion_zonas_metropolitanas_2010_Ca pitulos_I_a_IV>. Consultado el 27 de Julio de $201 \overline{4}$.

GARCÍA, Jaime. Análisis exploratorio de datos espaciales de la segregación urbana en Ciudad Juárez. Cuadernos de trabajo Universidad Autónoma de Ciudad Juárez, n. 2, p. $3-36,2011$.

HERNÁNDEZ, Myrna Limas. Geografía de la pobreza en Ciudad Juárez. Una perspectiva de género. Juárez: Universidad Autónoma de Ciudad Juárez, 2010.

HERNÁNDEZ, Vladimir. Análisis exploratorio espacial de los accidentes de tránsito en Ciudad Juárez, México. Revista Panamericana de Salud Pública, n. 5, p. 396 - 402, 2012.

JACQUEZ, Geoffrey; GREILING, Dunrie. Local Clustering in breast, lung and colorectal cancer in Long Island, New York. Journal of Health Geographic, v. 2, n. 3, p. $1-12,2003$.

SCOTT, Lauren; JANIKAS, Mark. Spatial Statistics in ArcGIS. In: FISCHER, Manfred; GETIS, Arthur. Handbook of Applied Spatial Analysis: Software tools, Methods and Applications. Berlin: Springer-Verlag, 2010 , p. $27-42$.

Recebido em 17 de dezembro de 2013. Aceito em 29 de maio de 2014. 